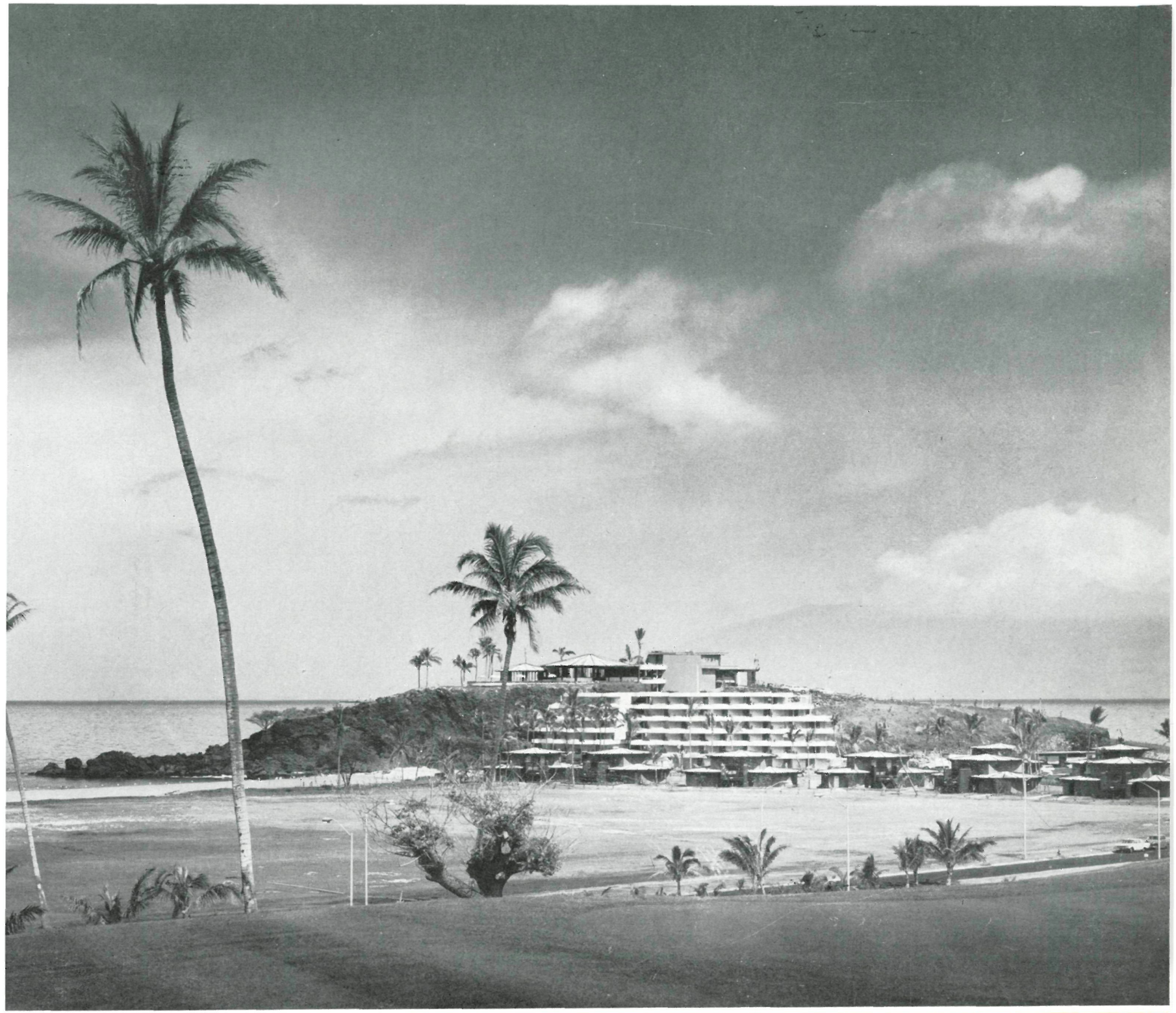

\title{
hotel Sheraton-Maui
}

en TRamapali, Mani Hawaii - U.S.A.

WIMBERLY, WHISENAND, ALLISON \& TONG, arquitectos

$147-27$

simopsis Las características que presentaba el terreno brindaron a los arquitectos la posibilidad de construir un hotel, que, además de crear un estrecho contacto con las bellezas naturales del emplazamiento, proporcionara a sus habitaciones una bella vista panorámica de la playa, la montaña y las islas adyacentes.

La entrada del tráfico rodado permite al visitante alcanzar la planta novena, que contiene: el bar, restaurante, vestíbulo, salas de relación, estar, etc.

Desde las plantas superiores se accede a los pisos donde están situadas las 72 habitaciones.

La «forma total» del edificio es una fiel expresión del logrado intento de integración del edificio con el terreno y el paisaje circundante. 

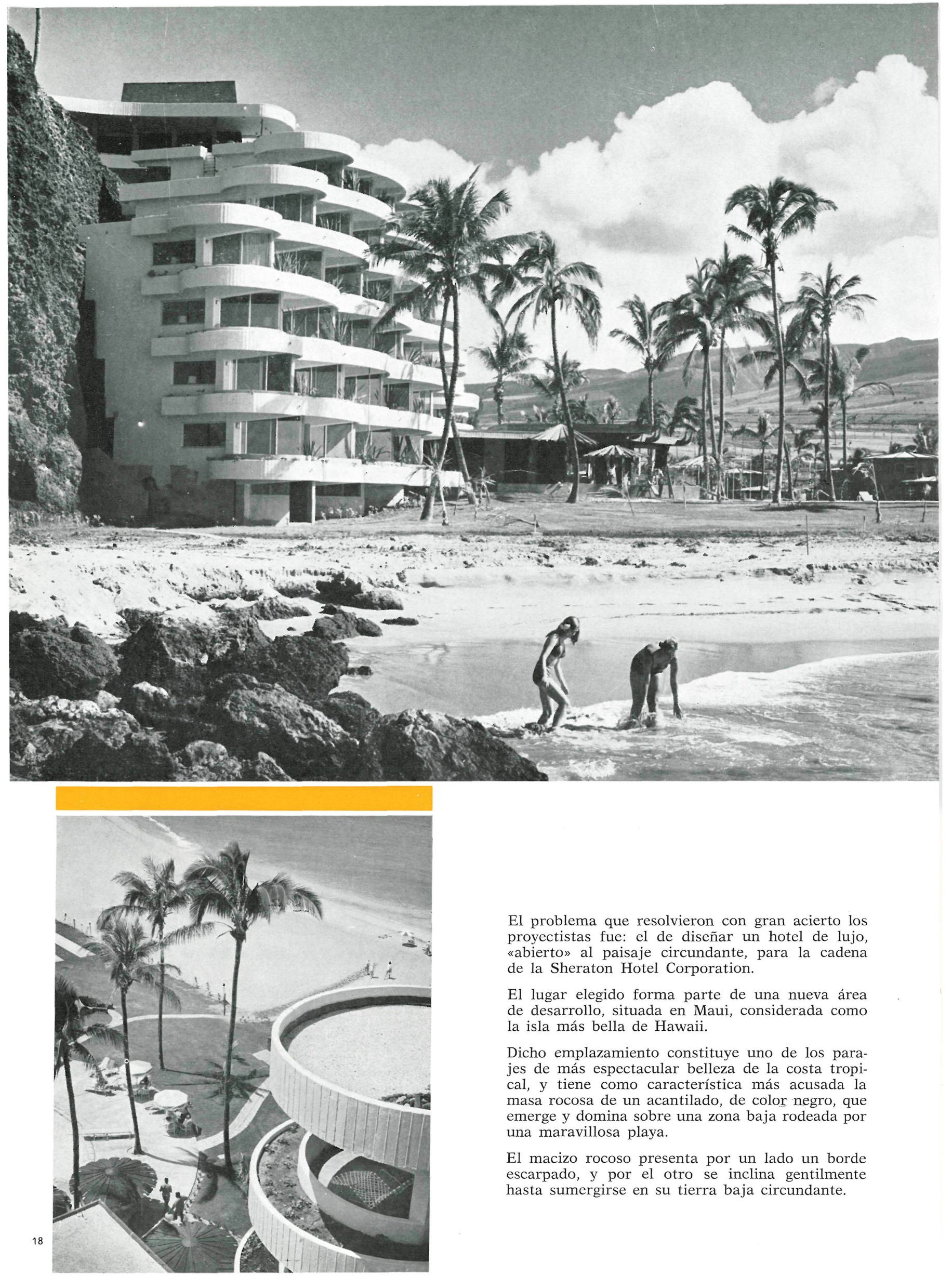

El problema que resolvieron con gran acierto los proyectistas fue: el de diseñar un hotel de lujo, «abierto» al paisaje circundante, para la cadena de la Sheraton Hotel Corporation.

El lugar elegido forma parte de una nueva área de desarrollo, situada en Maui, considerada como la isla más bella de Hawaii.

Dicho emplazamiento constituye uno de los parajes de más espectacular belleza de la costa tropical, y tiene como característica más acusada la masa rocosa de un acantilado, de color negro, que emerge y domina sobre una zona baja rodeada por una maravillosa playa.

El macizo rocoso presenta por un lado un borde escarpado, y por el otro se inclina gentilmente hasta sumergirse en su tierra baja circundante. 


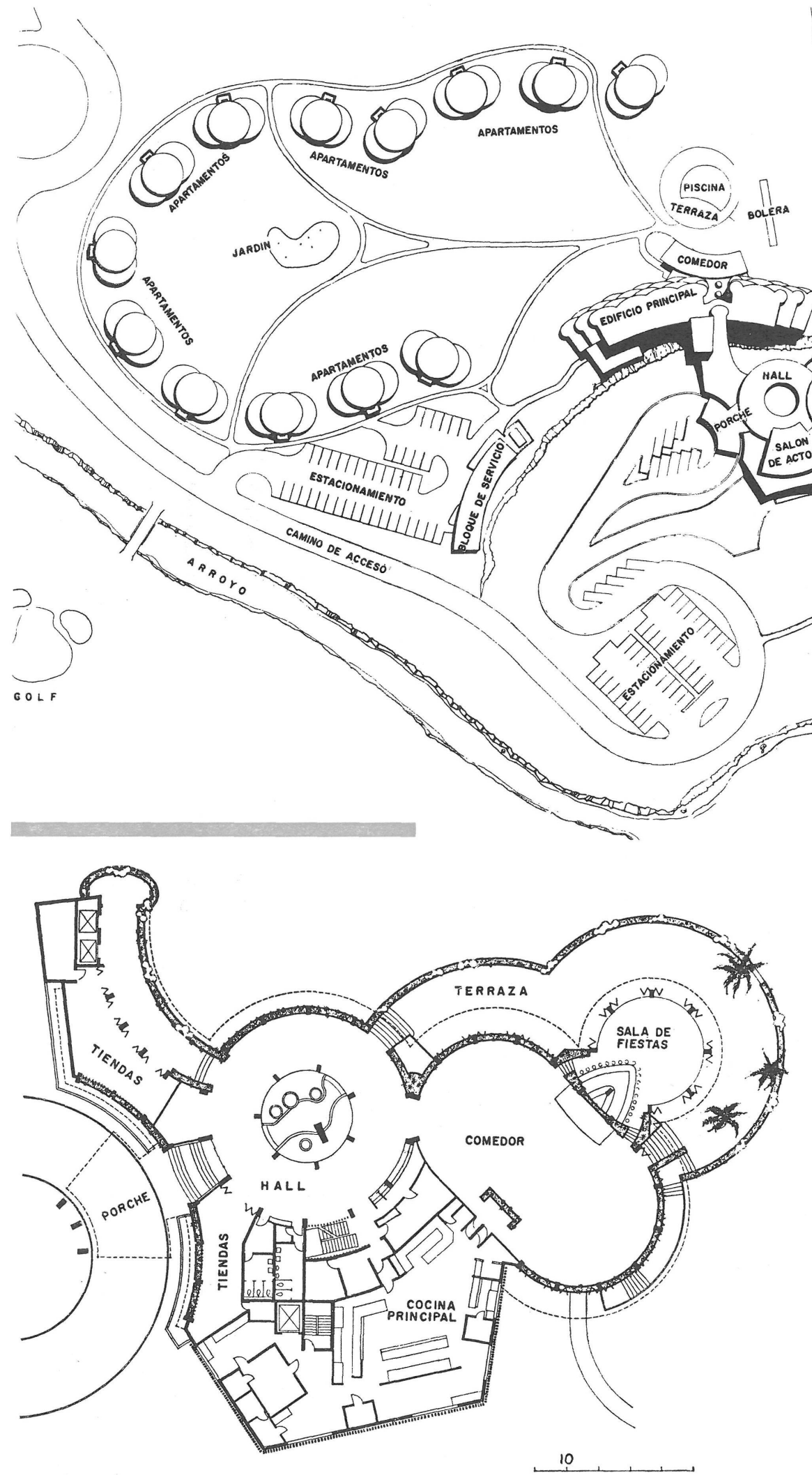

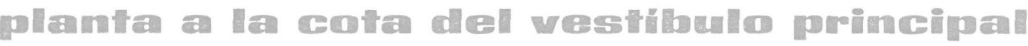

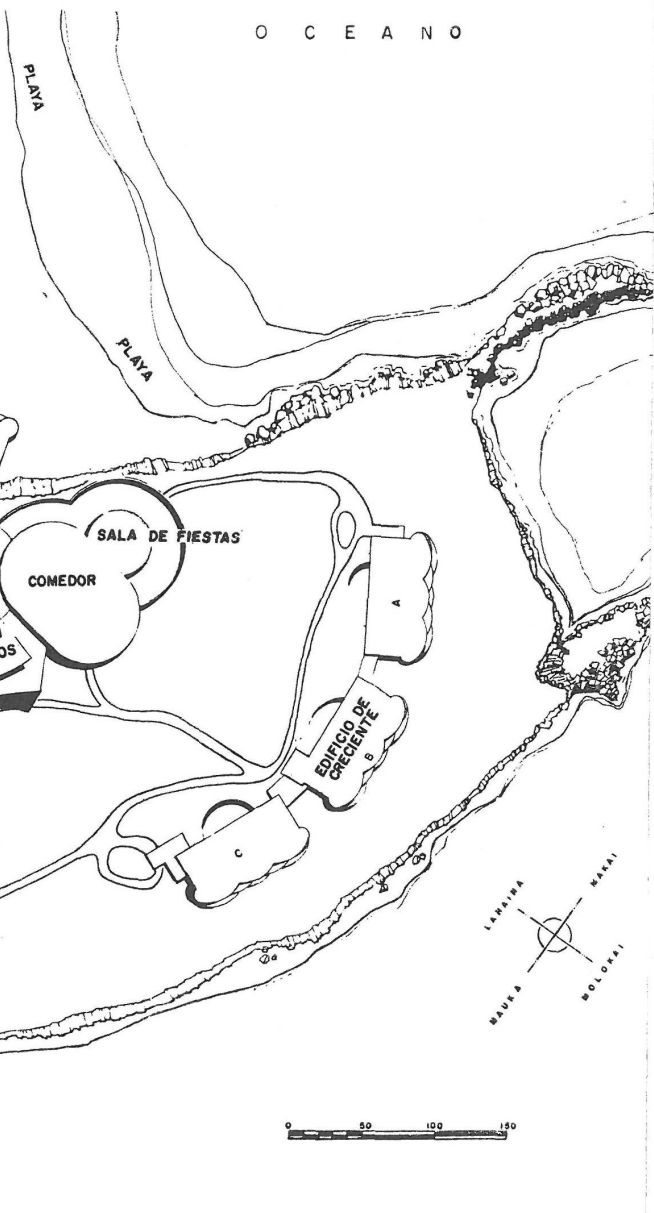

Estas características del terreno brindaron a los arquitectos la posibilidad de construir un hotel que, en estrecho contacto con las bellezas naturales del lugar, proporcionara a sus habitaciones una amplia vista dominando la playa, la montaña y las islas adyacentes.

Asimismo aprovecharon las oportunidades que ofrecía el escarpado peñón para integrarlo en un jardín que uniera la naturaleza con el edificio, lo que consiguieron incorporando a la frondosa vegetación una serie de jardines colgantes, situados por debajo de la vertiente escarpada del acantilado. Dichos jardines son obra del arquitecto paisajista George S. Walters. 
La entrada del tráfico rodado permite al visitante contemplar la parte posterior de la «Roca Negra» y alcanzar la planta novena, la cual contiene: el bar, restaurante, salas de relación social, etc.; cada una de ellas gozando de una apropiada orientación y con «vistas» a las islas adyacentes de Molokai y Lanai, así como a la iluminación nocturna de la histórica ciudad de Lahaina.

Desde las plantas superiores se desciende a los pisos donde están ubicadas las habitaciones. Por medio de un ascensor instalado en un hueco horadado en la roca, y atravesando el corredor existente en cada planta, se accede a una serie de terrazas situadas detrás de la superficie rocosa, y que, por la forma de haber sido tratadas paisajísticamente, producen en el espectador la sensación de encontrarse ante un jardín tropical adaptado en las rocas.

Cada una de las 72 habitaciones de la torre de ocho plantas está lo suficientemente vuelta hacia la parte posterior como para comunicarse con

uno de estos jardines tropicales, que proporcionan al conjunto el «verdor» adecuado para lograr integrar armónicamente el nuevo edificio en el paisaje natural que le rodea.

La silueta «total» del hotel es la expresión del intento de integrar el edificio al terreno; así, la «forma» que presenta el bloque de habitaciones está inspirada en la de las algas que recubren las zonas bajas de las formaciones coralíferas que abundan en la zona marina próxima; y la de las cubiertas de las habitaciones recuerdan a la de conchas marinas.

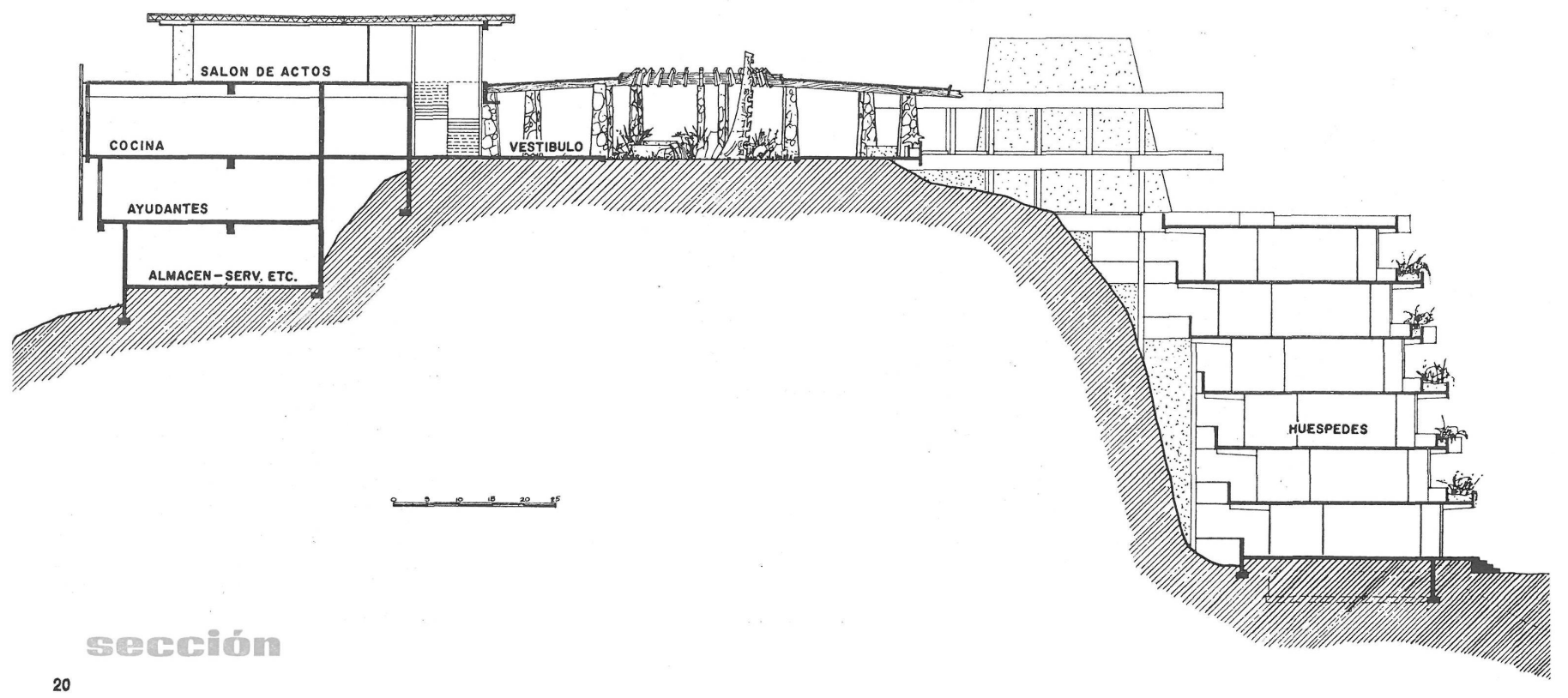




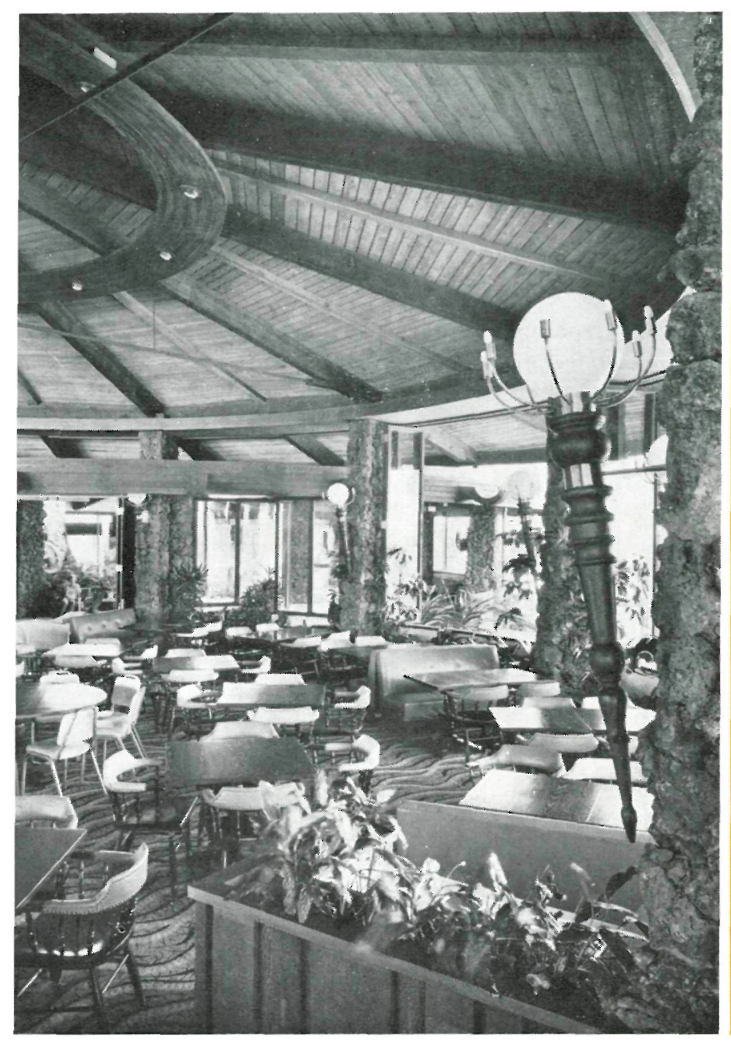

En definitiva, el edificio cumple satisfactoriamente, entre otros, los siguientes requisitos: goza de una excelente orientación respecto al sol y al viento; tiene una entrada al conjunto hotelero, de espectacular belleza; todas las habitaciones y zonas de relación social del hotel están dispuestas de manera que desde ellas se divisa una maravillosa vista panorámica de la playa y la flora tropical, y las luces de Lahaina (Maui) durante las horas nocturnas; se puede contemplar el paisaje, sin obstáculos, desde los jardines y el club adyacente; y presenta claramente separadas las zonas de baño de las destinadas para su empleo normal por el público.

La construcción ha sido realizada a base de hormigón armado; y la escalera que une el acantilado con el hotel propiamente dicho es también de hormigón armado y está decorada con motivos polinésicos.

Como integración de las artes plásticas a la Arquitectura, destacaremos: la fuente y el estanque labrado en piedra por el escultor Edward Malcolm Brownlee; en el comedor, el mural de Kipp Soldwedel, inspirado en una de las obras existentes en el New Bedford (Mass.) Whaling Museum, y en el bar, el motivo decorativo, obra de Benjamín Russell, inspirado asimismo en una de las obras existentes en el precitado museo.

Traducido y adaptado por J. M. Rubio

Fotos: R. WENKAM
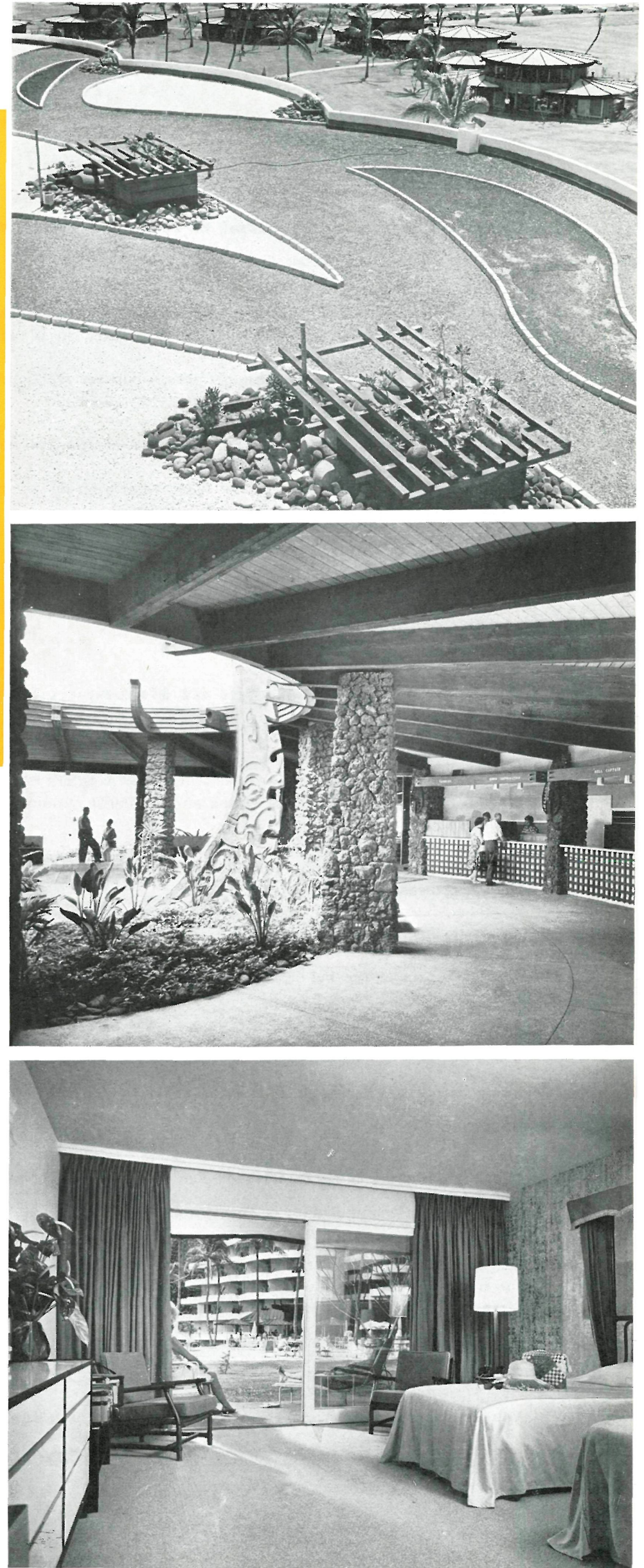


\section{Hôtrel Sheraton-Maui di Kacanapali, Maui Hawaii - Etats-Unis}

Wimberly, Whisenand, Allison \& Tong, architectes

Les caractéristiques du terrain ont permis aux architectes de construire un hôtel étroitement intégré aux beautés naturelles du site et doté de vues incomparables sur la plage, la montagne et les îles voisines.

L'entrée destinée aux automobiles permet au visiteur d'arriver jusqu'au neuvième étage abritant le bar, le restaurant, le vestibule, les salles de séjour, etc.

Des niveaux supérieurs on accède aux étages où se trouvent les 72 chambres.

La «forme totale» de l'édifice est une fidèle expression de la tentativ`, pleinement réussie, d'intégration de l'édifice avec le terrain et le paysage environnant.

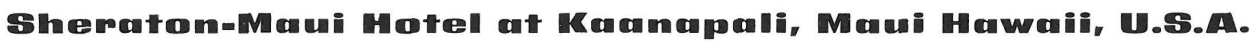

Wimberly, Whisenand, Allison \& Tong, architects

The topography of the site gave the designers the possibility of building an hotel which would establish an intimate link with the beauties of the surrounding environment and also provide wonderful views of the beach from the hotel rooms.

Cars can reach directly the ninth floor of the building, where the bar, restaurant, hall and social rooms of the hotel are located.

Access to the 72 hotel bedrooms is obtained directly from the top floors.

The total form of the building is a successful expression of the full integration of the hotel and the natural environment.

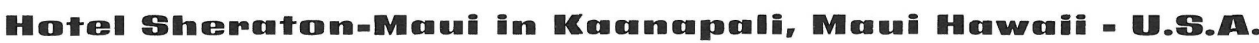

Wimberly, Whisenand, Allison \& Tong, Architekten

Die Beschaffenheit des Baugeländes gab den Architekten die Möglichkeit, ein Hotel zu errichten, das einen engen Kontakt mit den Naturschönheiten des Ortes herstellt und dessen Zimmer den herrlichen Blick auf den Strand, die Berge und die angrenzenden Inseln ermöglichen.

Von der Strasse aus gelangt der Besucher in das neunte Stockwerk, in dem die Bar, das Restaurant, Foyer, Gesellschaftsräume, usw. untergebracht sind.

Von den oberen Geschossen gelangt man in die unteren Stockwerke, in denen sich die y2 Zimmer befinden.

Der Versuch, das Gebäude harmonisch in das Gelände und die Landschaft einzugliedern, ist voll gelungen. 